\title{
The Recurrent Liver Disorder of a Pregnant Mother: Intrahepatic Cholestasis of Pregnancy - A Case Report and Literature Review
}

This article was published in the following Dove Press journal: International Medical Case Reports Journal

\author{
Animut Tagele Tamiru (D)' \\ Bayew Kelkay Rade (iD) \\ Oumer $\mathrm{Ali}^{2}$ \\ 'Department of General Midwifery, \\ School of Midwifery, College of Medicine \\ and Health Sciences Comprehensive \\ Specialized Hospital, University of \\ Gondar, Gondar, Ethiopia; ${ }^{2}$ Department \\ of Obstetrics and Gynecology, College of \\ Medicine and Health Sciences \\ Comprehensive Specialized Hospital, \\ University of Gondar, Gondar, Ethiopia
}

Correspondence: Animut Tagele Tamiru P.O Box: 196

Email animuut@gmail.com
Background: Intrahepatic cholestasis of pregnancy (ICP) is a form of liver disease which is unique to pregnancy with a worldwide prevalence ranging from $0.3 \%$ and $5.6 \%$ of pregnancies. It is presented with skin pruritus and elevated total serum bile salt and liver function test with unknown etiologic agent but suggested hormonal, environmental and genetic risk factors.

Case Presentation: A 31-year-old Gravida III and Para II mother came to University of Gondar specialized hospital at the outpatient clinic in January 2019 with complain of generalized pruritus along with jaundice at 24 weeks gestational age (GA). She presented with singleton and intrauterine pregnancy with a history of one neonatal loss, one living child, and elevated bilirubin, on admission blood serum test showed elevated serum transaminases, and bilirubin. At 30 weeks GA her bilirubin total and bilirubin direct tests were $4.52 \mathrm{mg} / \mathrm{dl}$ and $3.45 \mathrm{mg} / \mathrm{dl}$ respectively. At 34 weeks GA her bilirubin values became elevated. At 37 weeks GA fetal delivery was carried out via successful caesarean section with an indication of non-reassuring fetal heart rate pattern after induction with oxytocin. The outcome was stable for both mother and baby. After a two week follow up in the outpatient set up, her liver biochemistry test was normal, and free of the clinical features with normal physical growth and intact primitive reflexes of a newborn.

Conclusion: Presenting with a typical marker of increased liver function tests, bilirubin values and pruritus as a clinical feature, ICP was diagnosed. After an attempt at an oxytocin induction, an effective cesarean section was performed to deliver a live female baby, weighing $2.8 \mathrm{~kg}$. The case disappeared after three weeks follow up in the puerperium.

Keywords: cholestasis of pregnancy, pruritus, total bile acid, North West, Ethiopia

\section{Background}

As described by Ahlfeld (1883), intrahepatic cholestasis of pregnancy (ICP), is a frequent jaundice in pregnancy that can be relieved following delivery, it may reoccur in subsequent pregnancies. ${ }^{1}$ ICP is a common pregnancy-related liver disease seen in the second and third trimesters of pregnancy. ${ }^{2}$ Clinically it characterized by a rash and an itching sensation all over the body, particularly on the hands and feet. Elevated liver enzymes, including serum aminotransferases and/or elevated serum bile acid levels ( $>$ or $=10 \mathrm{micromol} / \mathrm{L}$ ) are usually spontaneously relieved after delivery, and no later than one month post-partum. ICP may reoccur in subsequent pregnancies. ${ }^{3}$ ICP is a liver disease unique to pregnancy with a global 
prevalence ranging from $0.3 \%$ and $5.6 \%$ of pregnancies. Its prevalence differs from one country to the other and is more common in countries like Chile and Bolivia. ${ }^{1,4}$

Even though, the pathogenesis of ICP is not well defined and its etiology is multifaceted, it is related to abnormal biliary transport across the canalicular membrane. Available literature suggest that genetic, environmental, hormonal, and exogenous factors all play a role in the occurrence of ICP. ${ }^{5-7}$ Even though ICP will not usually have severe and complex outcomes, it has been associated mostly with preterm delivery, meconium staining of amniotic fluid, fetal bradycardia, fetal distress and fetal demise. ${ }^{1,3,6,8-11}$ The underlying mechanisms associated with poor fetal outcome are largely unknown. Poor fetal outcomes, including asphyxia events and spontaneous preterm delivery, have been shown to be associated with elevated maternal total serum bile acids (TBA) (40 micromole/L) in pregnancy. ${ }^{3,12}$

It is controversial to set the standardized and the most optimal management for women with ICP. ${ }^{9}$ But pharmacotherapy, antenatal fetal monitoring, analysis of the bile acid and early elective delivery are the currently proposed management options, so as to reduce poor outcomes for both mother and baby. ${ }^{1,9,11,13}$

\section{Case Presentation}

A 31-year-old Gravida III and Para II mother came to the outpatient clinic of the University of Gondar specialized hospital, North West Ethiopia, in January 2019 complaining of pruritus (mainly under the breasts, on the neck, palms of the hands and soles of the feet) along with jaundice at 24 weeks gestational age (GA). She had a history of antenatal care follow up at a nearby health center. She presented to us with singleton and intrauterine pregnancy.

On arrival, she was screened for both subjective and objective data for her current and past obstetric, medical, surgical, gynecological, social, personal and family history. She had a history of early neonatal loss and one living child, her bilirubin value was elevated, she suffered pruritus and hepatomegaly in her previous pregnancies. She had a personal and family history of pruritus during pregnancy. From her previous personal history, she reported a history of similar features that resembled her current clinical presentation. The rest of her laboratory investigations and physical examination results, including vital signs (blood pressure 100/70 $\mathrm{mmHg}$ ), were in their normal range and she arranged for her next follow up after being provided with an antihistamine drug and offered counseling and health education to ensure the best outcome for her pregnancy. At 30 weeks GA, she was assessed for any complaints, including the worsening of pruritus and underwent liver biochemistry tests. Based on this, her bilirubin total and bilirubin direct tests were $4.52 \mathrm{mg} / \mathrm{dl}$ and $3.45 \mathrm{mg} / \mathrm{dl}$ respectively. Other complete blood count tests and urinalysis were within the normal range. The progress of the pregnancy was also assessed using ultrasound and showed no any abnormality. At 34 weeks GA her bilirubin values became elevated, whereas her liver function test on both alanine aminotransferase (ALT) and aspartate aminotransferase (AST) were 83U/L and $75 \mathrm{U} / \mathrm{L}$, respectively. The value of prothrombin time (PT) and partial thromboplastin time (PTT) were 12.2 and 34.6 seconds, respectively. Urine bilirubin, urobilinogen, urine nitrite, and Hepatitis B surface antigen (HBSantigen) tests were negative. But there was no opportunity for a TBA laboratory test.

As a result of having some abnormally elevated liver biochemistry tests, and the clinical features of the patient's current and past obstetric history, a decision was made to admitthe patient to the obstetric ward after a diagnosis of ICP was made. After admission various checks were carried out, including: weekly fetal surveillance with ultrasound and using a kick chart; administration of four doses of dexamethasone 12 hours apart to accelerate fetal lung maturation at 33 weeks GA; administration of antihistamine drugs to alleviate the suffering from pruritus; and psychological reassurance of the patient. The patient's clinical symptoms were not improved after administering antihistamine drugs and she suffered from severe pruritus following the administration of dexamethasone. At 37 weeks GA, the obstetrician and midwives had a detailed discussion and decided to deliver the baby. Initially the cervix was ripened with a Foley catheter so as to have an acceptable BISHOP score and then induction of labor with oxytocin was carried out. During this time a nonreassuring fetal heart rate pattern was detected with a cardiotocograph (CTG) and was confirmed with ultrasound. Finally, a successful caesarean section was done performed to deliver a $2.8 \mathrm{~kg}$ live female baby with an APGAR score of 8 and 9 in the 1st and 5th minutes, respectively. Following delivery, the patient remained inhospital for a week and was discharged to home with both mother and baby in stable conditions. The evaluation of the patient during the puerperium two weeks after giving birth was good, with the normalization of the liver 
biochemistry tests and the disappearance of the pruritus. The bilirubin total, ALT and AST decreased to $1.1 \mathrm{mg} / \mathrm{dl}$, $32 \mathrm{U} / \mathrm{L}$, and $31 \mathrm{U} / \mathrm{L}$ respectively. The evaluation of the baby was also good, with normal physical development.

\section{Discussion and Conclusion}

With the unknown etiology of ICP, various factors are indicated to be associated with high prevalence of ICP; these factors include genetics, the environment, coexisting liver and biliary tract conditions or abnormal metabolism of bile acid due to the high secretion of estrogen during pregnancy, hyper emesis gravidarum, multiple pregnancies and over stimulation of ovarian or oral contraception. The most frequent ICP complication to the fetus is preterm delivery. ${ }^{3,8}$ Especially the risk of preterm delivery is significantly higher for those patients with total bile acids (TBA) $>40 \mu \mathrm{mol} / \mathrm{l}^{3}{ }^{3}$ It was found that the mechanism of preterm delivery with ICP is that bile acid activity results in an increased sensitivity of the uterine muscle to oxytocin and in the increased oxytocin receptor expression. Having a TBA $>11 \mathrm{~mol} / \mathrm{L}$ in the third trimester of pregnancy is a direct indicative of ICP. The measurement of bile acid concentration is a basic test aimed at diagnosis and therapy monitoring of the ICP. Meanwhile, the activity of alcohol dehydrogenase (ADH) isoenzymes could be considered as having a positive interaction in the sera of women with intrahepatic cholestasis ${ }^{14}$ and having a history of allergic reactions may mean they are more likely to develop ICP ${ }^{15}$ but for our patient there was no history of allergic reactions. Hence it is better to consider such a test while suspecting and detecting this case. ICP has its own differential diagnoses including fatty liver disease, hepatobiliary disorder, HELLP syndrome, skin disease, renal pruritus and hyper emesis gravidarum. As a result, it is better to consider all these and differing diagnoses while anticipating ICP. In the management of ICP, the major role should be preventing still birth and minimizing the adverse effects of ICP clinical features on the mother. As various literatures suggest, ${ }^{8,11}$ there is no definitive cure for ICP other than alleviating the suffering from pruritus with drugs like Ursodeoxycholic acid (UDCA), ${ }^{2}$ antihistamines and delivering the baby as early as possible (from 37-38 weeks GA) as the clinical features of ICP will regress and disappear after delivery.

Finally, ICP is a cholestatic liver disease unique to pregnancy with a variable worldwide prevalence ranging between $0.3 \%$ and $5.6 \%$ of pregnancies. After confirming the diagnosis of ICP with a liver biochemistry test indicating total serum bile acid and its signs and symptoms as a clinical feature, close follow up of the patient is mandatory so as to prevent and minimize the adverse outcomes of ICP. For our patient after a serial laboratory test and weekly fetal surveillance, a trial of induction with oxytocin was performed and finally an effective cesarean section was carried out to deliver a $2.8 \mathrm{~kg}$ living female baby with an indication of non-reassuring fetal heart rate pattern. ICP regressed and disappeared at the three week follow up in the puerperium.

\section{Abbreviations}

ALT, alanine aminotransferase; AST, aspartate aminotransferase; GA, gestational age; ICP, intrahepatic cholestasis of pregnancy; PT, prothrombin time; PTT, partial thromboplastin time; TBA, total serum bile acid.

\section{Data Sharing Statement}

The data used to support the findings of this study are available from the corresponding author upon formal request.

\section{Ethical Approval and Consent to Participate}

The Ethical clearance letter was obtained from the Institutional Review Board of the University of Gondar. Patient consent was taken with written informed consent form and she was volunteer to participate.

\section{Consent for Publication}

Written informed consent was obtained from the patient for publication of this case report and any accompanying images.

\section{Acknowledgment}

We would like to acknowledge University of Gondar Department of Obstetrics and Gynecology as well as school of midwifery for allowing us to report on this case. Our deepest gratitude goes to our patient for her cooperation on revealing both her subjective and objective data, as well as her permission for us to publish this article.

\section{Author Contributions}

All authors made a significant contribution to the work reported, whether that is in the conception, study design, execution, acquisition of data, analysis and interpretation, 
or in all these areas; took part in drafting, revising or critically reviewing the article; gave final approval of the version to be published; have agreed on the journal to which the article has been submitted; and agree to be accountable for all aspects of the work.

\section{Disclosure}

The authors declared that they did not have any competing interest.

\section{References}

1. Ali Aya MK, Shazly SA, Abbas AM, Mohammed SA. Intrahepatic cholestasis of pregnancy. Evid Based Women's Health J. 2013;3(1-4).

2. Rodrigo Zapata F. Intrahepatic cholestasis of pregnancy: even today a puzzling disease of pregnancy. 2017.

3. Rook M, Vargas J, Caughey A, Bacchetti P, Rosenthal P, Bull L. Fetal outcomes in pregnancies complicated by intrahepatic cholestasis of pregnancy in a Northern California cohort. PLoS One. 2012;7(3): e28343. doi: 10.1371 /journal.pone. 0028343

4. Chacko KR, Wolkoff AW. Intrahepatic cholestasis of pregnancy: new diagnostic insights. Ann Hepatol. 2017;16(2):176-178. doi:10.5604/ 16652681.1231550

5. KondrackiEne JKL, Kupcinskas L. Intrahepatic cholestasis of pregnancy current achievements and unsolved problems. World $J$ Gastroenterol. 2006;14(38):5781-5788. doi:10.3748/wjg.14.5781
6. Shashank Shekhar S, Diddi G. Liver disease in pregnancy. Taiwan J Obstet Gynecol. 2015;54:475-482. doi:10.1016/j.tjog.2015.01.004

7. Li M. Recurrent intrahepatic cholestasis pregnancy: a case report. J Clin Obstet Gynecol Infertil. 2017;1(4):1018.

8. Kenyon AP, Piercy CN, Girling J, et al. Obstetric cholestasis, outcome with active management: a series of 70 cases. BJOG. 2002;109:282-288. doi:10.1111/j.1471-0528.2002.01368.x

9. Geenes V, Williamson LC, Chappell LC. Intrahepatic cholestasis of pregnancy. Obstetric Gynaecolog. 2016;18(4):273-281. doi:10.1111/ tog. 12308

10. Grone M AK, Smith JF. Intrahepatic cholestasis of pregnancy. Liver Dis. 2012;13(3).

11. Palmer KR, Xiaohua L, Mol BW. Management of intrahepatic cholestasis in pregnancy. Lancet. 2019;393(10174):853-854. doi:10.1016/S0140-6736(18)32323-7

12. A G, Marschall HU, Mattsson LA. Intrahepatic cholestasis of pregnancy: relationships between bile acid levels and fetal complication rates. Hepatology. 2004;40(2):467-474. doi:10.1002/hep.20336

13. Guideline RG-t. Obstetric cholestasis. Royal College of Obstetricians and Gynecologists; 2011:43.

14. Jelski W, Piechota J, Orywal K, Mroczko B. The alterations in alcohol dehydrogenase activity in the sera of women with intrahepatic cholestasis of pregnancy. Anticancer Res. 2020;40(4):1997-2001. doi:10.21873/anticanres.14155

15. Morton A, Laurie J. The biochemical diagnosis of intrahepatic cholestasis of pregnancy. Obstet Med. 2019;12(2):76-78. doi:10.1177/ $1753495 X 18795979$

\section{Publish your work in this journal}

The International Medical Case Reports Journal is an international, peer-reviewed open-access journal publishing original case reports from all medical specialties. Previously unpublished medical posters are also accepted relating to any area of clinical or preclinical science. Submissions should not normally exceed 2,000 words or 4 published pages including figures, diagrams and references. The manuscript management system is completely online and includes a very quick and fair peer-review system, which is all easy to use. Visit http://www.dovepress.com/testimonials.php to read real quotes from published authors. 